\title{
Characteristics and Relevant Factors of Sports Injury among Rural School-Aged Children in Zunyi, Southwest China: A Cross- Sectional Survey
}

\author{
Chan Nie ${ }^{1}$, Ya Hui ${ }^{1}$, Yanna Zhou ${ }^{1}$, Tao Wang ${ }^{1}$, Huiting Yu ${ }^{1}$, Shangpeng Shi ${ }^{1}$ and Xiuquan Shi, ${ }^{1,2 *}$ \\ ${ }^{1}$ Department of Epidemiology and Health Statistics, School of Public Health, Zunyi Medical University, China \\ ${ }^{2}$ The Research Institute at Nationwide Children's Hospital, The Ohio State University College of Medicine, USA
}

*Corresponding author: Xiuquan Shi, Department of Epidemiology and Health Statistics, School of Public Health, Zunyi Medical University, Zunyi, Guizhou 563006, China, E-mail:xqshi@zmc.edu.cn

\section{Introduction}

Sports injury is common among students, it occurs during sports or exercise activities such as football, basketball, volleyball. Most sports injuries affect the locomotor system, which are traumatic in most cases [1-3]. Although great attention has been paid to their prevention and treatment, injury prevention is still a great challenge in rural Zunyi [4], a low-income area in southwest China. We attempted to describe the characteristics of sports injury among school-aged children and reveal the related factors of it, so as to add some evidence for effective prevention measures.

\section{Methods and Results}

Injuries were defined if it met one of the following criteria: (1) Required medical attention or (2) Activities were restricted and rest was required for at least half a day because of sports injuries during the 12 months before the interview $[5,6]$. Using multistage cluster sampling, children age 8 to 15-years-old from 11 schools in Zunyi were recruited from June 2014 to July 2015. They were surveyed by designed questionnaires which consisted of five parts: demographic characteristics, family and school environment, sports injury situation and the KAP (Knowledge, Attitude, Practice) awareness in a recent year. Their information was collected, checked and then analyzed. The study was approved by the 11 targeted schools and the Institutional Review Board of Zunyi Medical University and the written informed consent was obtained by each student and their guardian(s). A database was constructed using the EpiData 3.1 (http://www.epidata.dk/). Data were double-entered to reduce errors. Analysis was performed using the statistical software package SPSS v18.0 (SPSS Inc., Chicago, IL, USA). P-value $<0.05$ and $95 \% \mathrm{Cl}$ of OR not contains 1 were considered statistically significant.

In the final, we surveyed 2217 children and retrieved 2127 eligible questionnaires (effective response rate $96.11 \%$ ). Of all the respondents, $53.1 \%$ (1130/2127) were boys and $46.9 \%$ (997/2127) were girls; $56.4 \%$ (1200/2127) were left-behind children (whose parents were away in cities, while their children were left behind to be cared for by a single parent or other family members) [7] and 43.6\% (927/2127) were nonleft-behind children. The annual incidence of sports injury was $26.52 \%$ among school-aged children in rural Zunyi. The main sport when injury happened was running (39.5\%), followed by playing soccer (20.9\%) and basketball (20.0\%). The leading injured body parts were knees (35.1\%), followed by ankles (24.5\%) and wrists (22.3\%). The types of children's sports injuries were mainly skin abrasions (41.1\%), followed by sprains (37.1\%), fractures (11.5\%), and other types of injuries (10.1\%). Additionally, most time of the injury was in the afternoon (41.8\%) and the most frequent place was at school (43.8\%).

According to the characteristics of the possible influencing factors, we divided the factors into sustained factors (such as gender, personality, age, left-behind or not, family income, KAP score) which were difficult or even cannot be changed; and instant factors (such as warm-up,

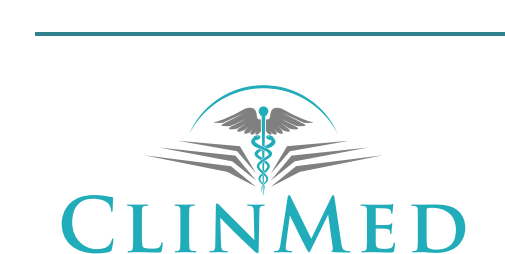

INTERNATIONAL LIBRARY

Citation: Nie C, Hui Y, Zhou Y, Wang T, Yu H, et al. (2017) Characteristics and Relevant Factors of Sports Injury among Rural School-Aged Children in Zunyi, Southwest China: A Cross-Sectional Survey. Int J Sports Exerc Med 3:076. doi.org/10.23937/2469-5718/1510076

Received: July 12, 2017; Accepted: September 20, 2017; Published: September 23, 2017

Copyright: (C) $2017 \mathrm{Nie}$ C, et al. This is an open-access article distributed under the terms of the Creative Commons Attribution License, which permits unrestricted use, distribution, and reproduction in any medium, provided the original author and source are credited. 
close care, sports site, floor types, shoes types) which were easy to be changed in a short time. The results showed the average level of OR value of instant factors was greater than that of sustained factors, the difference was statistically significant $(t=-2.328, P=0.040)$. Moreover, factors such as gender, personality, left-behind or not, parents' education level, KAP score, whether warm-up or not, sports site, sports time, shoes types were related to sports injury. The instant factors as unattended (do sports without anyone in charge) $(\mathrm{OR}=4.068,95 \%$ Confidence Interval [95\% $\mathrm{Cl}$ 3.368 7.744, $\mathrm{P}<0.001)$, without warm-up $(\mathrm{OR}=5.107$, $95 \% \mathrm{Cl} 3.368 \sim 7.744, \mathrm{P}<0.001)$, cement floor $(\mathrm{OR}=1.566$, $95 \% \mathrm{Cl} 1.047 \sim 2.343, \mathrm{P}=0.029$ ) were risk factors. Although the correct rate of children's knowledge of sports injury was $65.8 \%$ in total, only $30.9 \%$ and $21.1 \%$ of children had a right choice on the questions of "what to drink (tap-water, boiled water, lightly salted water or other beverages) after strenuous exercise" and "what time to warm up". Interestingly, we found that girls' KAP scores were a little higher than boys' $(4.67 \pm 1.14$ vs. $4.55 \pm 1.23, \mathrm{P}=0.019)$, while their injury incidence was obviously lower than boys' (20.7\% vs. $31.6 \%, \mathrm{P}<0.001)$.

\section{Conclusions}

From the results we conclude that pediatric sports injury is serious in rural Zunyi, southwest China, but children lack the related knowledge of how to prevent such injury. Closer supervision need to be taken by guardians or teachers when children do sports and health education should be provided to improve children's knowledge about sport-injury prevention. For example, guiding children to exercise at the right place, teaching them warm up before exercise and reminding them to wear proper shoes, etc., thereby reducing the incidence of pediatric sports injuries, really make the "sports health" $[1,8,9]$.

\section{Funding}

This project was funded by the National Natural Science Foundation of China (No. 81160350, PI: Xiuquan Shi). We gratefully thank all the investigators and participants who contributed to our study.

\section{Contributors}

XS conceived and designed the study, and made scientific comments and revisions. $\mathrm{CN}$ wrote the abstract. $\mathrm{YH}$ and SS gathered and analyzed the initial data. CN, TW, $\mathrm{YZ}$ and $\mathrm{HY}$ interpreted the data. All authors read and approved the final version of the manuscript for publication.

\section{Declaration of Interests}

No competing interests.

\section{References}

1. Radić B, Radić P, Duraković D (2014) Sports and health: equivalence or contrariety. Acta Clin Croat 53: 430-436.

2. Stracciolini A, Casciano R, Levey Friedman H, Meehan WP, Micheli LJ (2013) Pediatric sports injuries: an age comparison of children versus adolescents. Am J Sports Med 41: 19221929.

3. Stracciolini A, Casciano R, Levey Friedman H, Stein CJ, Meehan WP, et al. (2014) Pediatric sports injuries: a comparison of males versus females. Am J Sports Med 42: 965-972.

4. Shi XQ, Qi YH, Shi D, Yan C, Shi J, et al. (2014) Features and risk factors of nonfatal injury among the rural children: a survey of seven schools in a mountain area in Southwest China. PLoS One 9: e102099.

5. Wang H, Liu XX, Liu YX, Lin Y, Shen M (2011) Incidence and risk factors of non-fatal injuries in Chinese children aged 0-6 years: a case-control study. Injury 42: 521-524.

6. Zhu HP, Xia X, Xiang HY, Yu CH, Du YK (2012) Disability, home physical environment and non-fatal injuries among young children in China. PLoS One 7: e37766.

7. Shi S, Yang H, Hui Y, Zhou X, Wang T, et al. (2016) Epidemiologic characteristics, knowledge and risk factors of unintentional burns in rural children in Zunyi, Southwest China. Sci Rep 6: 35445.

8. Forjuoh SN (2015) Protecting children from injury. Int J Inj Contr Saf Promot 22: 1-2.

9. Rössler R, Donath L, Verhagen E, Junge A, Schweizer T, et al. (2014) Exercise-based injury prevention in child and adolescent sport: a systematic review and meta-analysis. Sports Med 44: 1733-1748. 